\title{
Visual preference in the four-month old infant
}

WILLIAM C. SPEARS ${ }^{2}$

UNIVERSITY OF DENVER

Four series of stimuli, varying in shape, or color, or in both dimensions, were presented according to the method of pair comparisons to separate groups of infants four months of age. Preference was measured in terms of relative visual fixation time. The shapes used differed in number of turns, area and contour being held constant, and hue, brightness and saturation were specified in Munsell coordinates. Little evidence was found for preference of one color to another, a!though there were indications that red and blue stimuli were preferred to a grey stimulus of the same shape. There was no demonstrated preference for any of the five regular polygonal shapes used. Where both shape and color were varied within a stimulus set, the ordering suggested dominance of choice by color.

In a previous study of the visual preference behavior of four-month-old infants, Spears (1964) found that, as measured by relative visual fixation time, red and blue stimuli were preferred to a similarly shaped grey stimulus, certain shapes or patterns were preferred to others, and shape dominated color as the basis for preference when members of a stimulus set varied in both color and shape. No completely consistent relation was obtained between the preference orderings for siape and amount of complexily, as defined by the amount of contour or the number of turns, or degree of symmetry, contour, however, being most consistent in this respect.

The present study was an attempt to evaluate the effects of varying the number of turns in a set of shapes, this variable, according to Attneave, being correlated with judgments of complexity (1957). Color, and area and contour, insofar as possible, were held constant. The five regular polygonal figures used-triangle, square, pentagon, hexagon and circle-necessarily varied in symmetry as well as in complexity. Each of the experimental groups viewed a different stimulus series, the stimuli being presented according to the method of pair comparisons and varying in shape, or color, or in both dimensions simultaneously. The four groups, designated by dimension (s) varied as Color (C), Shape (S), Shape-Color 1 (SC1) and Shape-Color 2 (SC2), were each made up of 10 infants around four months of age. About half of the Ss were obtained from a "Well-Baby Clinic" in Denver, the remainder from private homes and a nearby Catholic orphanage, the latter agency contributing about a half-dozen. The median age for all groups was approximately 145 days, the range being from 99 to 157 days.

Method

A cubical box, with interior surfaces finished in flat black, permitted the Ss to view pairs of stimulus cards at a distance of about 12 in. and $O$, by means of a one-way viewing screen, to observe and record the occurrences and duration of visual fixation of the pair components. Two Standard Electric clocks (time indicated to $1 / 100 \mathrm{sec}$.) were activated by separate pressure switches to accumulate time of fixation. Two fluorescent lamps (G。E.F14T12/CWX), mounted vertically and one to each side of the inner front side of the box, and baffled to prevent direct light from falling upon $S$, provided about 80 foot-candles of illumination at the stimuli, as determined by a G.E. 213 light meter. Room illumination was approximately 5 to 10 foot-candles. An experimental session consisted of 10 presentation trials of $15 \mathrm{sec}$. each, the beginning and ending of a trial being signalled by onset and offset of a signal light visible only to $O$. Accumulated clock readings were recorded to the nearest quarter second and stimuli changed during the $30 \mathrm{sec}$. intertrial period.

All stimulus cards had a base of white matte board 6 by $4-3 / 4$ in., and a viewable area of $4-3 / 4$ by 4-3/4 in. A stimulus set for each group consisted of five cards which produced the 10 different paircombinations seen by the group. Those for the Color group each consisted of two concentric circular rings, cut from one of the following Munsell papers: red $5 \mathrm{R} 5 / 6$, blue $5 \mathrm{~B} 5 / 6$, green $5 \mathrm{G} 5 / 6$, yellow $5 \mathrm{Y} 5 / 6$ and grey $\mathrm{N} / 5$. The Shape group viewed the combinations possible for the five polygonal figures, constructed like those of the Color group but all in Munsell paper $5 R$ 4/12. Areas for the Shape stimuli were all about $7 \mathrm{sq}$. in. with contour ranging from $34 \mathrm{in}$. for the triangle to $38 \mathrm{in.} \mathrm{for} \mathrm{the} \mathrm{circle.} \mathrm{Areas} \mathrm{and} \mathrm{contours}$ for the shapes used for SC1 and SC2 were similarly restricted. The former set was made up of two hexagonal shapes in $5 \mathrm{R} 5 / 6$ and $5 \mathrm{~B} 5 / 6$ and three square shapes in the same colors and $N / 5$ grey. For $\mathrm{SC} 2$, the two hexagonal shapes were in blue and grey and the three squares as in SC1. Letters were randomly assigned to the backs of the cards to permit "blind" selection by $O$ in accordance with a predetermined order of pair presentation. This sequence was chosen to minimize "time" and "space" errors and is that recommended by Ross (1939) as optimal in these respects. Odd-numbered Ss were given the sequence in one direction and even-numbered Ss the converse of that, with positions of pair components relative to each other reversed.

There were no major differences in the procedures used in the present and the earlier study. The infant having been positioned and seated on the supporting 
table with body and head facing the viewing opening and legs and feet extending into it, the viewing lights were turned on and the first pair of stimuli exposed by opening the viewing doors. At the end of $15 \mathrm{sec}$. the doors were closed for the $30 \mathrm{sec}$. intertrial period, with subsequent trials being administered in the same manner.

\section{Results}

Orderings based on the number of Ss preferring one member of a pair to another and over the 10 possible combinations in a set of stimuli are as follows: C group: Blue $=$ Red $>$ Green $>$ Yellow $>$ Grey; Shape group: Hex $=$ Pent $=$ Tri $>$ Cir $>$ Sq; SC1: Blue Hex = Red Hex $>$ Blue Sq $>$ Red Sq $>$ Grey Sq; and SC2: Red Hex $>$ Blue Sq $>$ Red $\mathrm{Sq}>\mathrm{G}$ rey $\mathrm{Sq}>\mathrm{Grey}$ Hex. Significant differences in preference for given stimuli, as evaluated by the sign test, and with $10 \mathrm{Ss}$, would be at the .02 and .002 level, according to whether 9 or 10 Ss preferred one member or another of a pair. The $\mathrm{C}$ and $\mathrm{S}$ groups produced no significant preferences and the SC1 group only one, the Red Hex over Grey Sq. SC2 produced four significant preferences, all at the .02 level, and as follows: Red and Blue Sq and Red Hex over Grey Sq, and Blue Sq over Grey Hex. Possible position bias was assessed in terms of $t$ values based on differences for each $S$ in a group in total time of fixation to the left and right. That for CS1 was significant at the .05 level.

The individual consistency of each $S$ was computed on the basis of the presence or absence of circular triads (Kendall, 1955), coeffecients of 1.00 being needed to indicate true ranking. Only two Ss in CS1 and four in CS2 produced such coefficients. Group agreement, as measured by the Kendall $u$, was significant at the .01 level for CS2 and the ordering of stimuli by that group can therefore be considered meaningful, at least as to preference order of stimuli in that set.

\section{Discussion}

As in the earlier study, there is little evidence for preference of one color to another. There is some evidence, particularly in the CS2 group, that colors, in this case red and blue, are preferred to grey. The position bias noted for SC1 may have washed out similar preferences in that group.

The lack of preference ordering obtained with the Shape group was disappointing and made it necessary to assign shapes to SC1 and SC2 on an arbitrary basis. Whether this may have led to the overall lack of significant or consistent results in these two groups is of course a matter of speculation. Only the SC2 subjects agreed significantly as to ordering of stimuli and here color seemed to govern choice. This is contrary to findings obtained with the SC1 group in the earlier study. There, the two shapes (bullseye and diagonal) and two colors (red and grey) used to make up the stimuli had been shown to differ significantly as regards preference and the ranking definitely indicated shape dominance. In a second group, however, SC2, the shapes used were a hexagonal and bullseye, and the colors, yellow and blue. These, for rational and empirical reasons, were presumed to offer a more difficult discriminative and preference problem, and, in fact, no significant preferences or ranking were found. Huang (1945), it may be noted, reported that the dominance of form or color was apparently related to the extent of dimensional difference obtaining in one or the other dimension.

The tendency to color dominance found in the present study may be the result, then, of the demonstrated lack of preference (and perhaps, discrimination) for the shapes used and the use of these shapes with colors, blue and red, which have generally been found to be preferred to a grey of equal brightness. If complexity is in fact related to the number of turns or angles in a figure, it would not appear to have been effective with the stimuli used. Symmetry would seem to have been equally ineffective. Perhaps, as Attneave suggests with respect to complexity, no single dimension or factor will be completely determinative of preference. If, as Birkhoff proposes (1933), preference is determined by the ratio of order and complexity, we may have to consider whether the stimuli in the present study possibly presented ratios sufficiently equivalent to inhibit preferential behavior.

\section{References}

Attneave, F. Physical determinants of the judged complexity of shapes. J. exp. Psychol., 1957, 53, 221-227.

Birkhoff, G. D. Aesthetic measure. Cambridge: Harvard University Press, 1933 .

Huang, I. Abstraction of form and color in children as a function of the stimulus object. J. genet. Psychol., 1945, 66, 59-62.

Kendall, M. G. Rank correlation methods. New York: Hafner, 1955.

Ross, R. T. Optimal orders in the method of pair comparisons. $J$. exp. Psychol., 1939, 25, 414-424.

Spears, W. C. Assessment of visual preference and discrimination in the fourmonth old infant. J. comp. physiol. Psychol., 1964, $57,381-386$.

\section{Notes}

1. This study was supported by Grant $\mathrm{MH}$ 08361-01 from the National Institute of Mental Health. The author wishes to thank Marian Senior, a graduate student at the University of Denver, for her competent running of the experiment and to express his appreciation to Mrs. Inez Alm and Dr. Chester Poremba of Children's Hospital, Denver, and to Mrs. Margaret Fletcher, of the Infant of Prague Nursery in the same city, for making subjects available. 2. Now at Queen's University, Kingston, Ontario. 